\title{
A Case Study in Distributed Locking Protocol on Linux Clusters
}

\author{
Sang-Jun Hwang ${ }^{1}$, Jaechun No ${ }^{1}$, and Sung Soon Park ${ }^{2}$ \\ 1 Dept. of Computer Software, \\ College of Electronics and Information Engineering, \\ Sejong University, Seoul, Korea \\ 2 Dept. of Computer Science \& Engineering, \\ College of Science and Engineering, \\ Anyang University, Anyang, Korea
}

\begin{abstract}
Today's scientific simulations often generate huge amounts of data for data archival, data analysis, and visualization. These data are stored in high-performance distributed storages that consist of a networkoriented computing environment. In such a computing environment, one of the major issues affecting in achieving substantial I/O performance and scalability is to build an efficient locking protocol. In this paper, we present a distributed locking protocol that enables multiple client nodes to simultaneously write their data to distinct data portions of a file, while providing the consistent view of client cached data, and conclude with an evaluation of the performance of our locking protocol on a Linux cluster.
\end{abstract}

\section{Introduction}

Today's scientific simulations often generate huge amounts of data for data archival, data analysis, and visualization, and then these data are stored in high performance distributed storages that consist of a network-oriented computing environment. The common network-oriented storage architecture designates a few number of network-attached servers as a data storage pool and connects the clients to the servers via network, like GigaEthernet or Fibre Channel [1, 2, 3, 5, 6]. In such a storage architecture, a critical issue affecting in achieving high I/O bandwidth and scalability for the scientific simulations is to build an efficient locking protocol which is used for providing the coordinated accesses to remotely stored data and for providing the consistent views of client cached data. The reason is that, in order to produce high I/O bandwidth, many scientific simulations use parallel I/O packages where multiple client nodes simultaneously perform their I/O operations. MPI-IO is among those parallel I/O packages. MPI-IO [8,10] is specifically designed to enable the optimizations that are critical for generating high-performance I/O. These optimizations include collective I/O and the ability to access noncontiguous data sets. However, in order to achieve high I/O performance using MPI-IO on a network-oriented distributed storage, the distributed file system which is running on top of the 
storage must provide the ability to lock a file per data section to have multiple concurrent writers. However, many of the locking protocols integrated with distributed file systems are based on a coarse-grained method [1, 2, 3] where only a single client at any given time is allowed to write its data to a file, while the other clients are waiting for the current node to finish its write operation even when the others would write to the different data portions of the same file. This drawback significantly degrades I/O performance in many scientific simulations where supporting parallel write operations happens to be proved generating high I/O bandwidth [4,7].

In this paper, we present a distributed locking protocol based on multiple reader/single writer semantics for a data portion to be accessed. In this scheme, a single lock is used to synchronize concurrent accesses to a data portion of a file. But, several nodes can simultaneously run on the district data sections in order to support data concurrency. We conclude our paper by discussing the performance evaluation of our locking protocol on a Linux cluster.

\section{Design Motivation}

Our main objectives in developing a distributed locking protocol were to provide high-performance parallel I/O, to minimize the communication latency occurred during the lock negotiation steps, and to utilize local lock services as much as possible.

- High-performance I/O We designed the distributed locking protocol capable of allowing multiple concurrent writers to the same file to achieve high performance I/O. Also, the locking protocol provides data consistency between the data stored in the storage device and the data stored in the client-side cache.

- Low communication latency We designed the locking protocol to reduce the network overhead taking place during the lock negotiation steps with Global Lock Manager (GLM). All the lock requests coming from the client nodes are evenly distributed on multiple GLMs. Moreover, in order to minimize the number of callback messages necessary to revoke and release a lock, we grouped all the client nodes into several node groups. If GLM finds the node group where the lock holder belongs to it then sends a lock revocation message to the node group.

- Use of local lock service we designed the locking protocol to utilize local lock service to the maximum extents in order not to incur communication overhead with GLM and remote lock holders. By retaining the privileges on data sections even in the absence of active processes on a client, we eliminated the need to communicate with GLM repeatedly for the same data section, and thus can minimize the network latency. 


\section{$3 \quad$ Implementation Details}

\subsection{Overview}

Figure 1 illustrates the distributed lock interface that is integrated with distributed file systems. Applications issue I/O requests using local file system interface, on top of VFS layer. Before performing an I/O request, each client should acquire an appropriate distributed lock from GLM in order to maintain data consistency between the cached data on clients and the remote, shared data on servers. The lock request is initiated by calling the lock interface, snq_clm_lock.

As mentioned in section 2 , in order to reduce the communication latency occurring in the lock acquire step, we grouped the client nodes into several node groups. In the current implementation, an eight bit integer is used to denote node groups. When a client acquires an appropriate lock to perform I/O operation, the bit corresponding to the node group where the client belongs to is set to 1 . Also, if a client requests a lock to GLM, GLM first locates the node group where the lock holder belongs to and then sends a callback message to the nodes of the node group. When the lock holder receives the callback message, it releases the requested lock and sends back an acknowledge to GLM to grant the lock to the requester.

Figure 2 represents a hierarchical overview of the locking construct with two client nodes and one GLM. The lock modes that we provide for are SHARED



Fig. 1. A distributed lock interface 




Fig. 2. A hierarchical overview of distributed locking protocol

for multiple read processes and EXCLUSIVE for a single write process. The lock structure consists of three levels: metalock, datalock, and childlock. The metalocks, inode 0 on node $\mathrm{A}$ and inode1 on node B in Figure 2, synchronize accesses to files and the value of a metalock is an inode number of the corresponding file. Below the metalock is a datalock responsible for coordinating accesses to a data portion. For example, on node A, metalock inode0 is split into two datalocks associated with the data sections 0-999 and 1000-1999 in bytes and, on node B, two datalocks below inode1 are associated with the data sections 0-2999 and 3000-5999 in bytes. In order to grant a datalock, the lock mode of the higher lock (metalock) must be SHARED, meaning that a file is shared between multiple clients.

The lowest level is a childlock that is of a split datalock. As mentioned in section 2, given that a datalock is granted, the datalock can be split further to maximize local lock services as long as the data section to be accessed by a requesting process does not exceed the data section of the datalock held. In other words, in Figure 2, the datalock for the data portion 0-999 is split into three childlocks that control accesses to the data portion 0-100, 100-199, and 800899, respectively. The childlock is locally granted and therefore the requesting process needs not communicate with GLM to obtain the childlock. However, the childlock is granted only when the lock mode of a childlock is compatible with that of the higher datalock. The datalock and childlock are found by comparing the starting file offset and data length being passed from the local file interface.

GLM contains the global lock information consisting of a list of locks that each GLM is responsible for serving. In Figure 2, GLM contains the metalocks, 
inode0 and inode1, and the datalocks of the data portions 0-999, 1000-1999, 02999, and 3000-5999 held by node A and node B. GLM also contains the node group information indicating those groups where the lock holders belong to.

\section{Performance Evaluation}

We measured the performance of the distributed locking protocol on the machines that have Pentium3 866MHz CPU, $256 \mathrm{MB}$ of RAM, and 100Mbps of Fast Ethernet. The operating system installed on those machines was RedHat 9.0 with Linux kernel 2.4.20-8. The performance results focused on the time to obtain locks by performing lock revoke, downgrade, and upgrade operations. The time to invalidate client cached data and to write dirty data to disk was not included in the evaluation.

Figures 3 and 4 represent the time to obtain the locks with the exclusive mode in write operations and with the shared mode in read operations, as the number of clients increases from 4 to 16. Also, in Figure 3, one machine was configured as a GLM and, in Figure 4, four machines were configured as GLMs. When four machines were configured as GLMs, each lock request is given to a GLM, according to round robin fashion. All clients read or wrote 1Mbytes of data to the distinct portions of the same file. In this case, the lock requested by each client is newly created on GLM and returned to the requesting client, causing no callback message to be sent to the remote lock holder. Figures 3 and 4 show that if there is no lock revocation occurred with the remote lock holder, then the communication overhead necessary for acquiring a new lock becomes

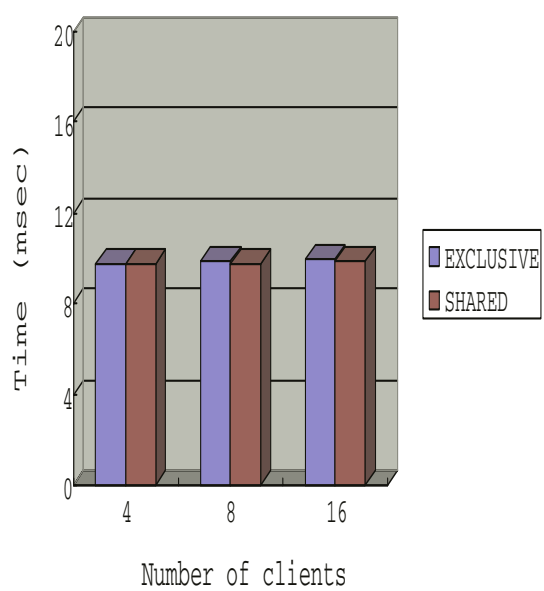

Fig. 3. Time overhead to acquire a distributed lock using one GLM. Each client read or wrote 1 Mbytes of data to the distinct section of the same file

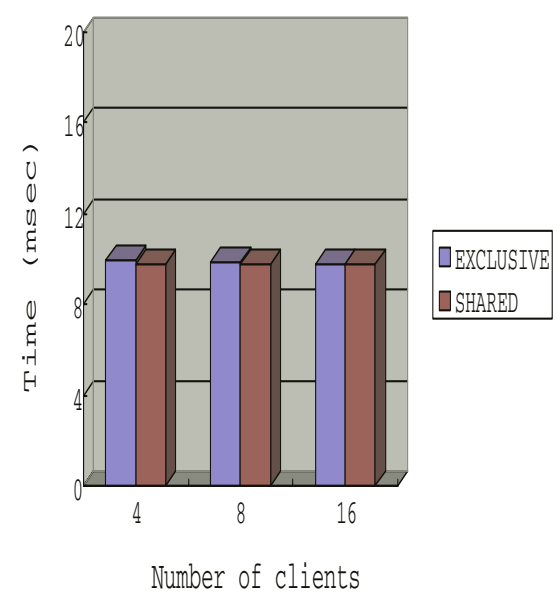

Fig. 4. Time overhead to acquire a distributed lock using four GLMs. Each client read or wrote 1 Mbytes of data to the distinct section of the same file 
small both with the exclusive mode and with the shared mode. Also, changing the number of GLMs from one to four doesn't affect the performance.

Figures [5] and [6] show the time to obtain the locks with the exclusive mode and with the shared mode, while moving each client's data section to access to the one given to the neighbor at the previous step. For example, at the first step, the first client accesses to the first 1Mbytes of data section of a file and the second client accesses to the second 1Mbytes of data section of the same file. At the second step, the first client's data section changes to the second 1Mbytes of data section for which the second client has already acquired the lock at the first step. Therefore, at the second step, the second client should yield the lock held to the first client, while taking a new lock from the third client. Also, Figure 5 shows the time taken by using one GLM and Figure 6] shows the time taken by using four GLMs.

Figures 5 and 6 both illustrate that the overhead of the lock revocation is significant with the exclusive mode because only a single client is allowed to write to a data section at any given time. With the shared mode, there is no need to contact the remote lock holder since a single lock can be shared between multiple nodes. With the shared lock mode, GLM just increases a counter denoting the number of shared lock holders before granting the lock. Finally, the communication overhead is decreased when the number of GLMs is changed from one to four since the lock requests issued by the clients can be distributed on the multiple GLMs.

In order to figure out how much the network latency occurred at the lock negotiation step dominates the performance, we changed the number of clients running on each node, while keeping the total number of clients as 16 . If the



Fig. 5. Time to acquire a distributed lock using one GLM. A client's data section is shifted to the one given to the neighbor at the previous step



Fig. 6. Time to acquire a distributed lock using four GLMs. A client's data section is shifted to the one given to the neighbor at the previous step 


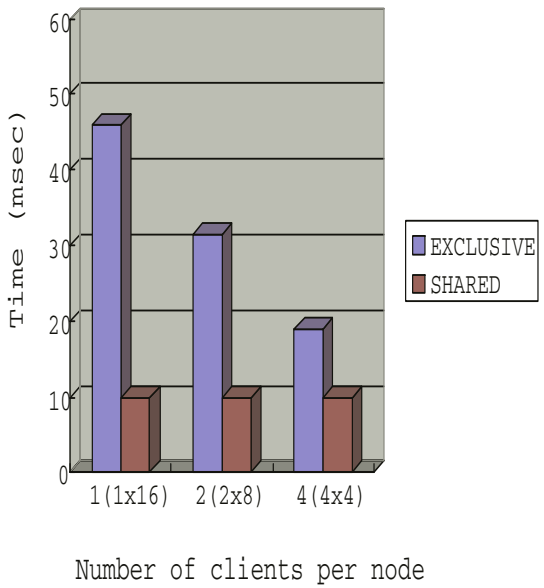

Fig. 7. Time overhead to acquire a distributed lock using one GLM as a function of number of clients running on each node. A client's data access range is shifted right at each step

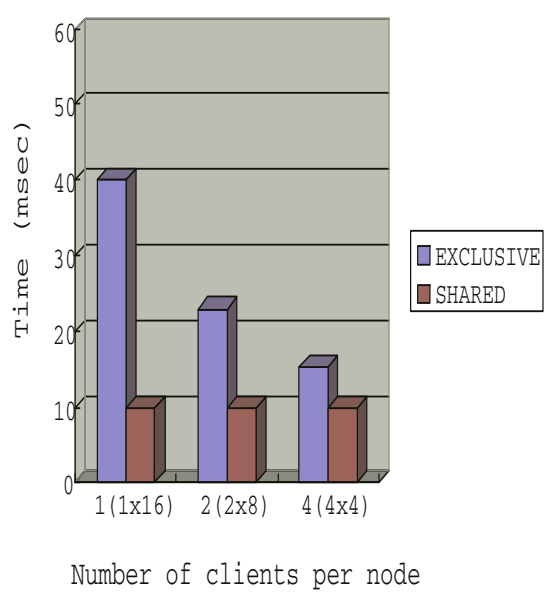

Fig. 8. Time overhead to acquire a distributed lock using four GLMs as a function of number of clients running on each node. A client's data access range is shifted right at each step

number of clients on a node is one, then every 16 clients runs on the different node. If the number of clients is two, then two clients run on each node and so 8 different nodes are needed to have 16 clients as total. Also, as did in Figures 5] and 6] we changed the data access range of each client to the one given to the neighbor at the previous step. With two clients running on the same node, the callback message is sent to the remote lock holder every two I/O operations to revoke a lock. With four clients, the callback message is sent to the remote lock holder every four I/O operations, resulting in the lock negotiation overhead decrement, compared to two clients on each node. According to this experiment, we could see that the dominating performance factor with 16 clients is the network overhead to contact the remote lock holder.

\section{Conclusion}

Concurrent accesses to the same file frequently occur in modern scientific simulations where allowing parallel write operations significantly improves I/O bandwidth. However, most distributed client-server file systems support a coarsegrained locking protocol in which all the concurrent write operations to a file are serialized even when the data sections being written are different between writers. In this paper, we presented a distributed locking protocol with which several nodes can simultaneously write to the distinct data portions of a file, while guaranteeing a consistent view of client cached data. The distributed locking protocol has also been designed to exploit locality of lock requests to minimize communication overhead with GLM and remote lock holders. 


\section{References}

1. Murthy Devarakonda, Bill Kish, and Ajay Mohindra. Recovery in the Calypso file system. ACM Transactions on Computer Systems, 14(3):287-310, August 1996

2. Chandramohan A. Thekkath, Timothy Mann, and Edward K. Lee. Frangipani: A Scalable Distributed File System. In Proceedings of the Symposium on Operating Systems Principles, 1997, pages 224-237

3. Kenneth W. Preslan, Andrew P. Barry, Jonathan E. Brassow, Grant M. Erickson, Erling Nygaard, Christopher J. Sabol, Steven R. Soltis, David C. Teigland, and Matthew T. O'Keefe. A 64-bit Shared Disk File System for Linux. In Proceedings of Sixteenth IEEE Mass Storage Systems Symposium Seventh NASA Goddard Conference on Mass Storage Systems \& Technologies, March 15-18, 1999

4. Jean-Pierre Prost, Richard Treumann, Richard Hedges, Bin Jia, Alice Koniges. MPI-IO/GPFS, an Optimized Implementation of MPI-IO on top of GPFS. In Proceedings of Supercomputing, November 2001

5. F. Schmuck and R. Haskin. GPFS: A Shared-Disk File System for Large Computing Clusters. In Proceedings of the First Conference on File and Storage Technologies(FAST), pages 231-244, Jan. 2002

6. P. J. Braam. The Lustre stroage architecture. Technical Report available at http://www.lustre.org, Lustre, 2002

7. Jaechun No, Rajeev Thakur, and Alok Choudhary. High-Performance Scientific Data Management System. Journal of Parallel and Distributed Computing, (64)4:434-447, April 2003

8. Jean-Pierre Prost. MPI-IO/PIOFS. World-Wide Web page at http://www.research.ibm.com/people/p/ prost/sections/mpiio.html, 1996

9. MacroImpact Inc., SANique CFS. A SAN Based Cluster File System, Version 2.1, Technical Report, August 2002

10. William Gropp and Ewing Lusk and Rajeev Thakur. Using MPI-2: A dvanced Features of the Message-Passing Interface, MIT Press, 1999, Cambridge, MA 